\title{
Investigation of Performance in Selected Auditory Tasks by Blind and Visually Impaired Children and Teenagers
}

\author{
E. Bogusz*, H. Koprowska And E. Skrodzka
}

\author{
Institute of Acoustics, A. Mickiewicz University, Umultowska 85, 61-614 Poznań, Poland
}

\begin{abstract}
The present work discusses results concerning sound perception obtained in selected auditory tasks, such as pitch discrimination and pitch-timbre categorization for blind and visually impaired subjects (children and teenagers). Listeners were divided into two age groups: 7-13 year olds and 14-18 year olds. The study tested 20 individuals ( 8 congenitally blind and 12 visually impaired) and 20 sighted persons comprising reference groups. The timing of the experiments was as short as possible due to the fact that our listeners were children. To date, no study has described results of such experiments for blind/visually handicapped children and teenagers. It was shown that the performance of blind/visually impaired participants was not as good as that of blind adults in pitch discrimination and pitch-timbre categorization tasks. These results may have implications for the development of auditory training in orientation and mobility of young visually handicapped people.
\end{abstract}

PACS: $43.66 . \mathrm{Hg}$

\section{Introduction}

A number of studies have shown that adult blind or visually handicapped people perform better than sighted individuals in tasks related to attention focusing [1], sound source localization [2], speech perception [3], word memorization [4], and pitch discrimination [1, 5]. Some papers indicate that congenitally blind or early-blind adult individuals display superior performance compared to late-blind persons $[1,5,6]$. The possible reasons for such behavior of early- and late blind persons are beyond the scope of this paper as they are of neuropsychological nature. In brief, it may be said that blind subjects (especially early blind) may be more susceptible to changes in brain function induced by blindness [5], e.g. for blind persons a larger tonotopic map in the auditory cortex was found, compared to the sighted reference group [7].

The present work discusses results concerning sound perception obtained in two selected psychoacoustic tasks, such as pitch discrimination and pitch-timbre categorization for blind or visually handicapped children and teenagers. Children and teenagers (blind/visually handicapped and normal sighted reference persons) were divided into two age groups: $7-13$ year olds and 14-18 year olds. Most of our subjects have been blind/visually handicapped since the first days of their life.

Our aim was to investigate selected auditory perception skills in blind and sighted children and teenagers, as no equivalent studies have been reported in literature yet. Additionally, we wanted to compare our results to those obtained for blind adults [5]. Thus, we wanted to check to what extent results recorded for younger and older

* corresponding author; e-mail: ebogusz@amu.edu.pl visually handicapped groups would be similar to the performance of blind adults, or whether the age of the blind individual influences the results of psychoacoustic tasks.

\section{Subjects}

The study was approved by the Bioethical Committee at the Poznań University of Medical Sciences. A total of 20 visually impaired persons and 20 sighted reference persons took part in the experiments. Table I lists demographic characteristics of blind/visually handicapped subjects. For each blind/visually handicapped participant a sighted control person was recruited, corresponding in terms of age, gender, preferred hand (right-handed people prefer the right ear, left-hand people prefer the left ear). All our subjects have had problems with vision ever since birth or soon after it, i.e. they were congenitally or early blind individuals. The early blind group comprised those who became blind after birth and before the 13th year of age. According to neuroimaging data [5, 8, 9], the late blind group comprises subjects who became blind at the age of 14 years or more. Consequently, none of our subjects was a late blind person. There was only one early blind subject. She/he was treated as a congenitally blind individual. Similarly, the only visually handicapped subject having sight problems from the age of 4 was treated as visually impaired from birth.

All the subjects were volunteers, they were not paid for their cooperation and they had no previous experience in psychoacoustic experiments. The visually impaired subjects were recruited from the Special Training and Education Centre for Blind Children in Owińska, Poland. None of the subjects had any significant hearing loss (as assessed by a tonal audiogram), and they were all free of neurological effects. They could stop tests at any time if they felt tired or uncomfortable. 
TABLE I

Characteristics of the blind/visually handicapped group. $\mathrm{C}$ - congenitally blind, $\mathrm{E}$ - early-onset, $\mathrm{V}-$ visually handicapped.

\begin{tabular}{c|c|c|c|c|c}
\hline \hline Subject & Age of blindness onset [yrs] & Age at test [yrs] & Age group & Gender & Preferred ear \\
\hline C_1 & 0 & 12 & $7-13$ & male & left \\
C_2 & 0 & 7 & $7-13$ & male & right \\
C_3 & 0 & 10 & $7-13$ & female & right \\
C_4 & 0 & 10 & $7-13$ & female & right \\
C_5 & 0 & 15 & $14-18$ & female & left \\
C_6 & 0 & 15 & $14-18$ & female & right \\
C_7 & 0 & 16 & $14-18$ & male & left \\
E_1 & 4 & 16 & male & right \\
V_1 & 0 & 13 & $7-13$ & female & left \\
V_2 & 0 & 13 & $7-13$ & male & right \\
V_3 & 0 & 13 & $7-13$ & female & left \\
V_4 & 0 & 12 & $7-13$ & female & right \\
V_5 & 0 & 9 & $7-13$ & female & right \\
V_6 & 0 & 10 & $7-13$ & female & right \\
V_7 & 4 & 7 & $7-13$ & female & right \\
V_8 & 0 & 15 & $14-18$ & male & right \\
V_9 & 0 & 15 & $14-18$ & male & right \\
V_10 & 0 & 15 & $14-18$ & male & right \\
V_11 & 0 & 17 & $14-18$ & female & left \\
V_12 & 0 & 15 & $14-18$ & male & left
\end{tabular}

TABLE II

Parameters of the pitch-timbre categorization experiment.

\begin{tabular}{c|c|c|c|c}
\hline \hline & Low pitch, soft & Low pitch, bright & High pitch, soft & High pitch, bright \\
\hline$f_{0}[\mathrm{~Hz}]$ & 294 & 294 & 417 & 417 \\
harmonic numbers & $3,4,5$ & $4,5,6$ & $3,4,5$ & $4,5,6$
\end{tabular}

TABLE III

Statistical significance of differences in means between groups of tested subjects in the pitch discrimination task.

\begin{tabular}{|c|c|c|c|}
\hline \multirow[t]{2}{*}{ Compared groups } & \multicolumn{3}{|c|}{ Frequency difference } \\
\hline & $0.5 \%$ & $1 \%$ & $2 \%$ \\
\hline $\begin{array}{l}\text { Younger blind subjects and } \\
\text { younger sighted reference group }\end{array}$ & & & $t_{(4,0.013107)}=4.254878$ \\
\hline $\begin{array}{l}\text { Older blind subjects and older } \\
\text { visually impaired subjects }\end{array}$ & $t_{(7,0.046681)}=2.411358$ & & \\
\hline $\begin{array}{l}\text { Younger blind subjects and older } \\
\text { blind subjects }\end{array}$ & $t_{(5,0.022902)}=-3.24186$ & & $t_{(5,0.024272)}=-3.18974$ \\
\hline $\begin{array}{l}\text { Younger visually impaired subjects } \\
\text { and older visually impaired subjects }\end{array}$ & & $t_{(10,0.026541)}=-2.59892$ & \\
\hline
\end{tabular}


TABLE IV

Statistical significance of differences in means between tested subjects and congenitally blind adults in the pitch discrimination task.

\begin{tabular}{|c|c|c|c|c|}
\hline \multirow[t]{2}{*}{ Compared groups } & \multicolumn{4}{|c|}{ Frequency difference } \\
\hline & $0.25 \%$ & $0.5 \%$ & $1 \%$ & $2 \%$ \\
\hline $\begin{array}{l}\text { Younger blind subjects } \\
\text { and blind adults }\end{array}$ & $p=0.0032$ & $p=0.0011$ & $\begin{array}{l}\text { No statistical } \\
\text { difference }\end{array}$ & $\begin{array}{c}\text { No statistical } \\
\text { difference }\end{array}$ \\
\hline $\begin{array}{l}\text { Older blind subjects } \\
\text { and blind adults }\end{array}$ & $p=0.0071$ & $\begin{array}{l}\text { No statistical } \\
\text { difference }\end{array}$ & $\begin{array}{l}\text { No statistical } \\
\text { difference }\end{array}$ & $\begin{array}{l}\text { No statistical } \\
\text { difference }\end{array}$ \\
\hline $\begin{array}{l}\text { Younger visually impaired } \\
\text { subjects and blind adults }\end{array}$ & $p=0$ & $p=0.0036$ & $p=0.009$ & $p=0.0065$ \\
\hline $\begin{array}{l}\text { Older visually impaired } \\
\text { subjects and blind adults }\end{array}$ & $p=0.0059$ & $p=0.0014$ & $\begin{array}{l}\text { No statistical } \\
\text { difference }\end{array}$ & $\begin{array}{l}\text { No statistical } \\
\text { difference }\end{array}$ \\
\hline
\end{tabular}

TABLE V

Statistical significance of differences in means between groups of tested subjects in the pitch-timbre categorization task.

\begin{tabular}{l|c|c}
\hline \hline \multirow{2}{*}{ Compared groups } & \multicolumn{2}{|c}{ Condition } \\
\cline { 2 - 3 } & No difference in pitch and timbre & Difference in timbre only \\
\hline Younger blind subjects and younger & $t_{(6,0.4110)}=3.421941$ & \\
sighted reference group & & $t_{(6,0.003445)}=4.666283$ \\
Older blind subjects and older sighted & & $t_{(7,0.018761)}=3.043245$ \\
reference group & &
\end{tabular}

TABLE VI

Statistical significance of differences in means between tested subjects and congenitally blind adults in pitch-timbre categorization task.

\begin{tabular}{l|c|c|c|c}
\hline \hline \multicolumn{1}{c|}{ Compared groups } & \multicolumn{4}{|c}{ Condition } \\
\cline { 2 - 5 } & $\begin{array}{c}\text { No difference } \\
\text { in pitch and timbre }\end{array}$ & $\begin{array}{c}\text { Difference } \\
\text { in timbre only }\end{array}$ & $\begin{array}{c}\text { Difference } \\
\text { in pitch only }\end{array}$ & $\begin{array}{c}\text { Difference in both } \\
\text { pitch and timbre }\end{array}$ \\
\hline $\begin{array}{l}\text { Younger blind subjects and } \\
\text { blind adults }\end{array}$ & $\begin{array}{c}\text { No statistical } \\
\text { difference } \\
\text { No statistical } \\
\text { difference }\end{array}$ & $p=0.0036$ & $\begin{array}{c}\text { No statistical } \\
\text { difference } \\
\text { Older blind subjects and } \\
\text { blind adults }\end{array}$ & $p=0.0023$ \\
$\begin{array}{l}\text { Younger visually impaired } \\
\text { subjects and blind adults } \\
\begin{array}{l}\text { Older visually impaired } \\
\text { subjects and blind adults }\end{array}\end{array}$ & $p=0$ & $p=0.0008$ & $p=0$ \\
\end{tabular}




\section{Experiments}

Two experiments that assessed different aspects of auditory perception were performed: pitch discrimination and pitch-timbre categorization. The experiments were similar to those performed by Wan et al. [5]. Sounds were prepared before the experiments in the Matlab environment. All the tasks were based on the experimental method of constant stimuli. The sounds were presented in a random sequence in both experiments. The timing of the experiments was as short as possible due to the fact that some of our subjects were children below ten years of age. Both experiments were presented via headphones to the preferred listener's ear chosen in relation to the preferred hand. Subjects were given a practice session prior to testing. They answered to experimental tasks verbally and their answers were copied by the experimenter.

\subsection{Pitch discrimination}

The pitch discrimination experiment was similar to the paradigm of Wan et al. [5] and Bonnel et al. [10]. The task of the subjects was to say which sound in a presented pair of sounds was higher in pitch. The choice of experiment parameters was typical for pitch discrimination tasks, i.e. each trial contained two $300 \mathrm{~ms}$ tones. The duration of $300 \mathrm{~ms}$ is long enough to evoke pitch sensation [11]. An attack and decay rate was $20 \mathrm{~ms}$ for each signal. They were much longer from the time constants of auditory filters which may play a role in the discrimination task [12]. The time interval between two tones in a pair was $300 \mathrm{~ms}$. It was long enough to avoid both backward and forward masking [12]. After each pair of signals there was an interval of $4 \mathrm{~s}$ for giving an answer. Signals were presented in a random order. Each pair contained a tone of frequency $f(500,750,1000$, or $1500 \mathrm{~Hz}$ ) and another tone of frequency either $2 \%, 1 \%$, $0.5 \%$, or $0.25 \%$ higher than frequency $f$. Additionally, in the practice session frequencies higher than frequency $f$ of $8 \%$ and $4 \%$ were used to teach subjects what a pitch discrimination task is. For adult subjects with normal hearing just a noticeable difference in pitch is related to a $0.2-0.3 \% f$ discrepancy from frequency $f$ in the range of $500-1500 \mathrm{~Hz}$ [12]. Therefore, we expected that frequency discrimination for pairs $(f, f+0.25 \% f)$ might be difficult for the youngest subjects. However, we decided to use the smallest frequency changes to be consistent with the experiment of Wan et al. [5].

Signals were presented at a sound pressure level comfortable to the subjects. Each pair of tones, e.g. $(f, f+$ $1 \% \mathrm{f}$ ) was presented in 5 repetitions, resulting in a total of 160 trials. The total duration of the experiment including breaks was about 30-40 min.

\subsection{Pitch-timbre categorization}

The pitch-timbre categorization experiment was adopted from the experiments of Wan et al. [5] and
Pitt [13]. Signals containing four tones of equal amplitude (basic frequency and three harmonics) were presented simultaneously. The signals had two levels of pitch and two levels of timbre. Pitch was determined by the frequency of the first tone $f_{1}: 294 \mathrm{~Hz}$ (for low pitch) or $417 \mathrm{~Hz}$ (for high pitch). Timbre was determined by manipulating harmonics of the first frequency $f_{1}$. Signals of frequency $f_{1}$ and harmonics $3 f_{1}, 4 f_{1}$ and $5 f_{1}$ had softer or darker timbre. Signals of frequency $f_{1}$ and harmonics $4 f_{1}, 5 f_{1}$ and $6 f_{1}$ had sharper or brighter timbre [14].

Parameters of the signals used in the pitch-timbre categorization experiment are listed in Table II. Each trial contained four tones lasting $300 \mathrm{~ms}$ with $20 \mathrm{~ms}$ of the attack and decay rate. The time interval between two signals in a pair was $1500 \mathrm{~ms}$. After each pair of signals there was an interval of $5 \mathrm{~s}$ for giving an answer. The signals were presented in a random order. The task of the subjects was to assess each pair of signals in both pitch and timbre. They were asked to say whether the signals were similar in pitch and timbre, and if not - which feature was different. Possible answers were: no difference in pitch and timbre, difference in pitch only, difference in timbre only, difference in both pitch and timbre. Each pair of signals was presented in 5 repetitions. The total duration of the experiment was about $25 \mathrm{~min}$.

\section{Results}

Similarly to Wan et al. [5], proportion correct was a primary dependent variable for all the experiments. For each of the tasks (pitch discrimination and pitch-timbre categorization) a question similar to Wan et al. [5] was asked: is there any advantage of the blind/visually handicapped over sighted subjects and if so, does it vary for the two age groups and for the difficulty level?

To evaluate the differences in means between any two groups the $t$-test for independent variables was used. The equality of variances assumption was verified with the Levene and Brown-Forsythe test. There were no statistically significant differences in variances in any compared pair of groups.

\subsection{Pitch discrimination}

Four difficulty levels were investigated: $0.25 \%, 0.5 \%$, $1 \%$, and $2 \%$ difference between tone frequencies. Results of the pitch discrimination experiment are shown in Fig. 1a, c, e (left column of Fig. 1) for both age groups of congenitally blind subjects, both age groups of visually impaired subjects and blind and visually impaired subjects, respectively. Results obtained for corresponding reference groups of sighted individuals are shown, too. Empty circles in Fig. 1 indicate results recorded for congenitally blind adults obtained by Wan et al. [5]. Error bars in all presented figures indicate standard errors of means. A statistical significance in means revealed for tested groups of subjects in the pitch discrimination task is shown in Table III. There were no significant statistical differences in means for remaining compared groups for remaining frequency differences. 

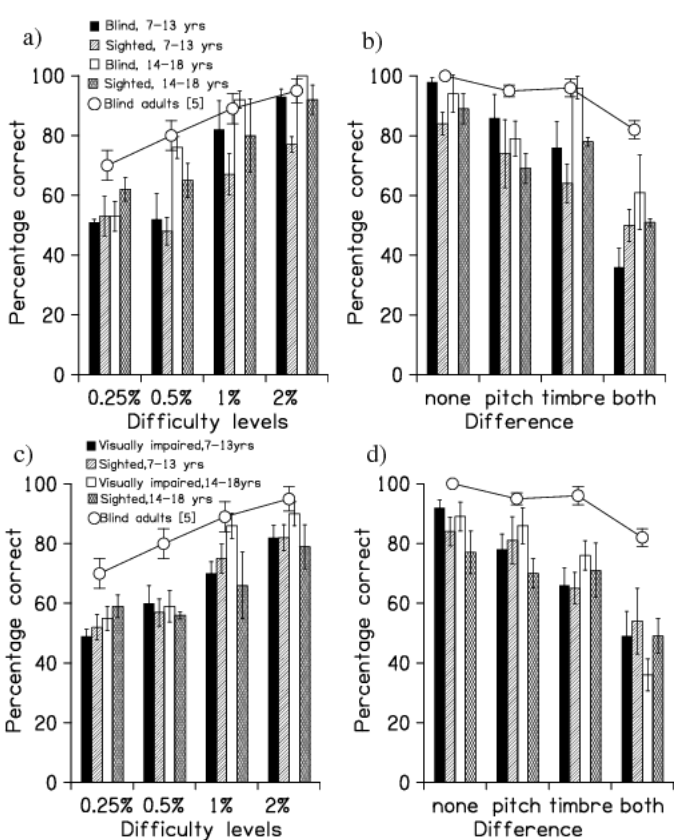

d)
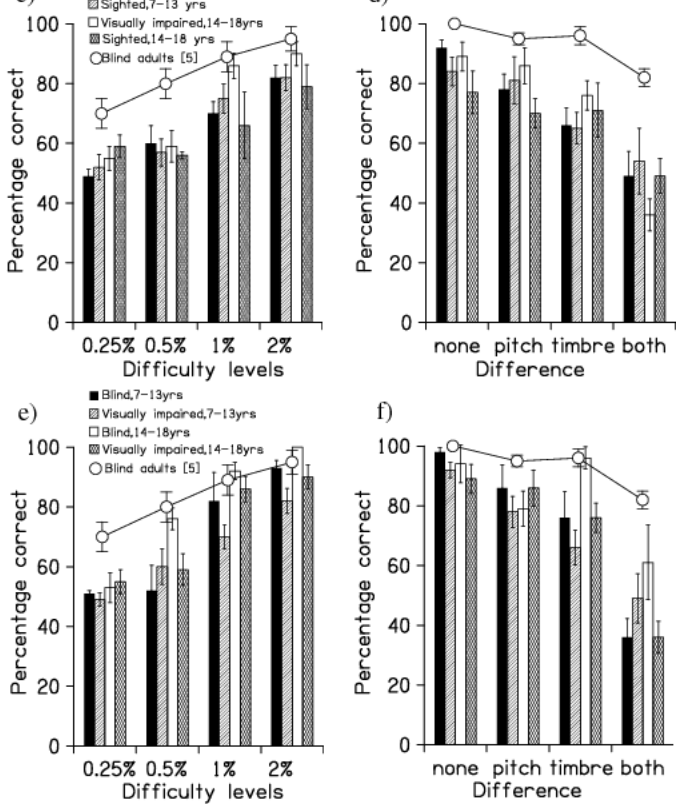

Fig. 1. Mean and standard errors of percentage correct in: (a) the pitch discrimination task for both age groups of blind subjects and corresponding reference groups. (b) The pitch-timbre categorization task for both age groups of blind subjects and corresponding reference groups. (c) The pitch discrimination task for both age groups of visually impaired subjects and corresponding reference groups. (d) The pitch-timbre categorization task for both age groups of visually impaired subjects and corresponding reference groups. (e) The pitch discrimination task for both age groups of blind subjects and both age groups of visually impaired subjects. (f) The pitch-timbre categorization task for both age groups of blind subjects and both age groups of visually impaired subjects. Empty circles indicate results for congenitally blind adults obtained by Wan et al. [5].

Results obtained for blind and visually impaired children (younger group) and teenagers (older group) were compared to results obtained for congenitally blind adults reported by Wan et al. [5]. The $t$-test of difference between means of two independent samples was used because means and standard errors were available for congenitally blind adults. A statistical significance in means revealed for compared groups of subjects in the pitch discrimination task is shown in Table IV.

\subsection{Pitch-timbre categorization}

Four conditions were used: no difference in pitch and timbre, difference in pitch only, difference in timbre only and difference in both pitch and timbre. Results of the pitch-timbre categorization experiment are shown in Fig. 1b, d, f (right column of Fig. 1) in the same manner as results of the pitch discrimination experiment. A statistical significance in means was revealed for pairs of tested groups shown in Table V. Differences between the remaining groups for remaining conditions were not statistically significant.

Results of blind and visually impaired children and teenagers were compared with results obtained for congenitally blind adults reported by Wan et al. [5]. The $t$-test of means of two independent samples was used. A statistical significance in means revealed for compared groups of subjects in the pitch-timbre task is shown in Table VI.

\section{Discussion}

Results from the pitch discrimination experiment are not homogeneous for all four difficulty levels/four frequency differences, i.e. they do not show the superior performance of blind subjects or visually impaired participants over sighted persons. Among blind participants, only the younger group obtained better results than its reference group for the easiest task when the frequency difference between two tones was $2 \%$.

When blind and visually impaired subjects were compared, it was found that the older group of blind individuals outperformed the blind group of children for the frequency differences of $0.5 \%$ and $2 \%$. The blind group of teenagers obtained better results than the impaired group of teenagers for the frequency difference of $0.5 \%$, too. The group of visually impaired teenagers had a better performance than the group of impaired children for the frequency difference of $1 \%$. The frequency difference of $0.5 \%$ is a difference slightly over just noticeable differences in pitch for adult sighted persons with normal hearing. This "threshold task" appeared to be difficult for blind children who had not acquired so much experience in auditory tasks as blind teenagers.

When results of the pitch discrimination task for children and teenagers were compared to results of congenitally blind adults, the pattern was more homogeneous. For the frequency difference of $0.25 \%$ correct answers oscillated around the chance level (50\% of correct) for all our subjects and were significantly lower from the results of blind adults. For the remaining frequency differences the older group of blind subjects did not differ significantly from the group of adults. The older group of visually impaired listeners did not differ significantly from the group of adults for the two highest frequency differences - $1 \%$ and $2 \%$. Thus, the blind teenagers achieved the level of blind adults for all perceptible frequency differences, while the group of blind children needs more time and practice. Visually impaired participants performed 
worse than their blind counterparts. The younger group of them did not obtain results similar to the blind adults for any frequency difference. The older group performed similarly to blind adults in the two easiest tasks. Visually impaired subjects probably rely on their residual sight and do not pay as much attention to sounds as blind persons.

In the pitch-timbre categorization experiment the blind group of children performed better than its reference sighted group for the easiest condition of "no difference in pitch and timbre" and the older blind group had a higher percentage of correct answers than its reference group for the condition "difference in timbre only". Blind teenagers outperformed visually impaired teenagers for the same condition. Consequently, blind participants performed better in the easy tasks.

The results of blind and visually impaired children and teenagers were then compared to the results of blind adults. For the most difficult condition of "difference in both pitch and timbre" the group of blind adults outperformed all our subjects. Both age groups of visually impaired individuals performed worse than blind adults for the condition "no difference in pitch and timbre". Results similar to blind adults were obtained in both age groups of blind people for the condition "no difference in pitch and timbre", the group of blind teenagers for the condition "difference in timbre only" and groups of visually impaired children and blind teenagers for the condition "difference in pitch only". Thus, out of total sixteen chances ( 4 conditions, 2 blind groups, 2 impaired groups) our subjects performed similarly to blind adults in five only.

This is the first study to evaluate the performance of blind and visually impaired children and teenagers as separate groups and compare them to congenitally blind adults. Our results show that blindness or visual impairment does not necessary lead to the superior performance in all auditory tasks and that congenitally blind adults may be more sensitive to differences in frequency parameters of auditory stimuli. Proper and correct distinction of those is extremely important for safe and independent orientation and mobility of visually handicapped people in the urban environment.

\section{Conclusions}

We conclude that:

- In the pitch discrimination and pitch-timbre categorization tasks some differences and similarities were found in performance between our subjects themselves (blind and visually impaired children and teenagers) as well as between our subjects and congenitally blind adults.
- In the most difficult trials in the pitch discrimination experiment (frequency difference between two tones as small as $0.5 \%$ ) and in the pitch-timbre categorization experiment (conditions "changes in both pitch and timbre" and "changes in timbre only") blind teenagers performed much better than the remaining three groups of our subjects, but still worse than congenitally blind adults for the condition "changes in both pitch and timbre".

\section{Acknowledgments}

We are very indebted to the teachers and pupils of the Special Training and Education Centre for Blind Children in Owińska, Poland for their help, work, cooperation and patience during the experiments.

We are also much grateful to the two anonymous reviewers for their helpful comments and suggestions.

\section{References}

[1] F. Gougoux, F. Lepore, M. Lassonde, P. Voss, P.R.J. Zatorre, P. Belin, Nature 430, 309 (2004).

[2] N. Lessard, M. Pare, F. Lepore, M. Lassonde, Nature 395, 278 (1998).

[3] K. Hugdahl, M. Ek, F. Takio, T. Rintee, J. Tuomainen, C. Haarala, H. Hämäläinen, Cognitive Brain Research 19, 28 (2004).

[4] A. Amedi, N. Raz, P. Pianka, R. Malach, E. Zohary, Nature Neurosci. 6, 758 (2003).

[5] C.Y. Wan, A.G. Wood, D.C. Reutens, S.J. Wilson, Neuropsychology 48, 344 (2010).

[6] P. Voss, F. Gougoux, R. Zatorre, M. Lassonde, F. Lepore, Neuroimage 40, 746 (2008).

[7] T. Elbert, A. Sterr, B. Rockstroh, C. Pantev, M.M. Muller, E. Taub, J. Neurosci. 22, 9941 (2002).

[8] L.G. Cohen, R.A. Weeks, N. Sadato, P. Celnik, K. Ishii, M. Hallett, Ann. Neurol. 45, 451 (1999).

[9] N. Sadato, T. Okada, M. Honda, Y. Yonekura, Neuroimage 16, 389 (2002).

[10] A. Bonnel, L. Mottron, I. Peretz, M. Trudel, E. Gallun, A.M. Bonnel, J. Cognitive Neurosci. 15, 226 (2003).

[11] E. Ozimek, Sound and Perception, PWN, Warszawa 2002 (in Polish).

[12] B.C.J. Moore, An Introduction to the Psychology of Hearing, 1st ed., PWN, Poznań 1999 (in Polish).

[13] M.A. Pitt, J. Exp. Psychol-Human Percept. Perform. 20, 976 (1994).

[14] G.E. Starr, M.A. Pitt, J. Acoust. Soc. Am. 102, 486 (1997). 